bei unzureichender Pufferkapazität ist bei $\mathrm{pH} 9$ größer als bei $\mathrm{pH} 7,6$.

Aufgrund der vorliegenden Untersuchungen kann die Bestimmung der durch GSH aktivierten Serum-CPK über die Vorwärtsreaktion im optischen Test als die am wenigsten störanfällige Methode mit einer auch für Normalseren ausreichenden Empfindlichkeit angesehen werden.

\title{
Literatur
}

1. Aebi, U., R. Richterich, J. P. Colombo und E. Rossi, Enzymol. biol. clin. 1, 61 (1961/62). - 2. Askonas, B. A., Biochem. J. 48, 42 (1951). - 3. BaumanN, P., J. Escher und R. Richterich, Schweiz. Zschr. Sportmed. 10, 33 (1962). - 4. Bernt, E. und H. U. BergmeYer, in: H.-U. Bergmeyer, Methoden der enzymatischen Analyse, S. 859. Verlag Chemie GmbH, Weinheim/Bergstraße (1962). - 5. BURger, A., R. RichTERICH und H. AEBr, Biochem. Z. 339, 305 (1964). - 6. Chappell, J. B. und S. V. Perry, Biochem. J. 57, 421 (1954). 7. Соlombo, J. P., R. Richterich und E. Rossr, Klin. Wschr. 40, 37 (1962). - 8. Conn, R. B. und V. Anrdo, Amer. J. Clin. Path. 46, 177 (1966). - 9. Deul, D. H. und J. F. L. van Breemen, Clin. chim. Acta (Amsterdam) 10, 276 (1964). - 10. Dreyfus, J.-C., G. SChaptra und J.DÉmos, Rev. franc. Et.clin.biol.5,384 (1960). - 11. Dreyfus, J.-C. und G. Schapira, Rev. franc. Et. clin. biol. 5, 386 (1960). - 12. Dreyfus, J.-C. und G. Schapira, Rev. franc. Et. clin. biol. 6, 700 (1961). - 13. Duma, R. J., und A. L. Sieger, Arch. Int. Med., Chicago 115, 443 (1963). - 14. Ebashr, S., Y. Toyokura, H. Momor und H. Sugrta, J. Biochem. (Tokyo) 46, 103 (1959). - 15. ENNOR, A. H. und H. RosenBerg, Biochem. J. 57, 203 (1954). - 15. Fiske, C. H. und Y. Subbarow, J. biol. Chemistry 81, 629 (1929). - 17. Fleisher, G. A., W. M. McConAHEY und M. Pankow, Mayo Clin. Proc. 40, 300 (1965). - 18. Graig, F. A. und G. Ross, Metabolism 12, 57 (1963). - 19. Graig, F. A. und J. C. Smith, J. clin. Endocr., Springfield 25, 723 (1965). - 20. Griffiths, P. D., Lancet (London) $I, 894$ (1963). - 21. Hugres, B. P., Clin. chim. Acta (Amsterdam) 7, 597 (1962). 22. Jacobs, H., H. W. Heldt und M. Klingenberg, Biochem.
Biophys. Res. Comm. 16, 516 (1964). - 23. KAR, N. C. und C. M. Pearson, Amer. J. Clin. Path. 43, 207 (1965). - 24. Kuby, S. A., L. Noda und H. A. Lardy, J. biol. Chemistry 209, 191 (1954). 25. Lehimann, F.-G., K. W. Schnemer und H. Menge, Enzymol. biol. clin. 6, 36 (1966). - 26. Nielsen, L. und B. Ludvigsen, J. Laborat. Clin. Med., S. Louis 62, 159 (1963). - 27. NodA, L., T. NiheI und M. F. Morales, J. biol. Chemistry 235, 2830 (1960). - 28. Okinaka, S., H. Sugita, H. Momor, Y. Toyokura, H. KuMagat, S. Ebashr und Y. Fujre, 84th. ann. Meet. Amer. neurol. Ass., Atlantic City (1959). - 29. Olrver, I. T., Biochem. J. 61, 116 (1955). - 30. Rosakr, S. B., Nature (London) 207, 414 (1965). - 31. Rotthauwe, H. W., S. Zurukzoglu-Sklavounou und H. HaMManN, Klin. Wschr. 39, 1269 (1961). - 32. Rotrhauwe, H. W. und M. Cerqueiro, Klin. Wschr. 41, 876 (1963). - 33. Rotrhauwe, H. W. und M. Cerqueiro, Clin. chim. Acta (Amsterdam) 10, 134 (1964). - 34. Rotrhauwe, H. W. und S. KowaLEWSKr, Klin. Wschr. 43, 150 (1965). - 35. RotrhauWe, H. W. und S. KowalewsKr, Humangenetik 3, 30 (1966). - 36. RotTHaUwe, H. W. und S. Kowalewiski, Klin. Wschr., 45, 387 (1967). - 37. SAX, S. M. und J. J. Moore, Clin. Chem. (New York) 11, 951 (1965). - 38. Schapira, F. und J.-C. Dreyfus, Ann. Biol. clin. 22, 349 (1964). - 39. SJövall, K. und A. Vorgr, Nature (London) 202, 701 (1964). - 40. SteIN, P. und W. LAMPrecht, Klin. Wschr. 40, 177 (1962). - 41. TANZER, M. L. und C. Gilvarg, J. biol. Chemistry 234, 3201 (1959). - 42. WEBER, H. und R. Richterich, Klin. Wschr. 41, 665 (1963). - 43. Wrison, K. M., K. A. Evans und C. O. Carter, Brit. Med. J. 1965, 750.

Priv.-Doz. Dr. Dr. H. W. Rotthauve 53 Bonn, Adenauerallee 119

\section{Dünnschichtchromatographische Methode zur İsolierung und Bestimmung der Porphyrine im Urin}

\author{
Von M. Doss und W. MaNNHEIM \\ Aus dem Hygiene-Institut der Universität Marburg/Labn (Direktor: Prof. Dr. R. Siegert)
}

(Eingegangen am 17. Januar 1967)

Es wird eine quantitative Methode für die Analyse von Copro- und Uroporphyrin im Urin beschrieben. Sie besteht darin, daß nach Lyophilisierung des Urins Porphyrinmethylester hergestellt, dünnschichtchromatographisch isoliert und in Chloroform spektrophotometrisch gemessen werden.

A quantitative method is reported for the analysis of copro- and uroporphyrin in urine. After lyophilisation of the urine, porphyrin methyl esters are prepared, isolated by thin layer chromatography and measured in chloroform solution.

Porphyrine werden im allgemeinen als freie Säuren in wäßriger Salzsäure oder als Methylester in organischen Lösungsmitteln photometrisch bestimmt (1-5). Die Isolierung der freien Säuren erfolgt mit Adsorptionsund Extraktionsmethoden sowie mit säulenchromatographischen und papierelektrophoretischen Trennungsgängen (Zusammenstellung bei (1)). Die Porphyrinextrakte enthalten meist noch Verunreinigungen, so daß ihre quantitative Bestimmung durch Messung der Absorption in verschiedenen Wellenlängen und mit Hilfe von Korrekturfaktoren durchgeführt wird $(6-8)$.

Bei der Untersuchung des Porphyringehalts von Bakterienkulturfiltraten zeigte sich, daß kleinste Mengen von Protoporphyrin ( $<1 \mathrm{nMol} / \mathrm{m} l)$ nach direkter Veresterung der Porphyrine im biologischen Material zu Methylestern und anschließender dünnschichtchromato- 
graphischer Auftrennung und Isolierung rein dargestellt werden können (9). Eine qualitative chromatographische Trennung von Porphyrinmethylestern auf Kieselgel wurde bercits von Demole (10) angegeben. Die präparativen und analytischen Möglichkeiten eines dünnschichtchromatographischen Verfahrens sind jedoch nicht nähcr untersucht und genutzt worden. Ihre Prüfung wurde durch die Tatsache angeregt, daß die im UV-Licht bei $355 \mathrm{~nm}$ rotfluoreszierenden Porphyrinmethylester bei der Extraktion aus der Methanol/ $\mathrm{H}_{2} \mathrm{SO}_{4} /$ WasserPhase schnell und vollständig in Chloroform überführt werden können. $\mathrm{Da}$ sich die Methylester der einzelnen Porphyrine im Gegensatz zu den freien Säuren in ihrer Löslichkeit wenig unterscheiden, bietet sich die Chromatographie als geeigneteste Trennungsmethode an. Bei der routinemäßigen Untersuchung bakterieller Porphyrine ergab sich, daß die Dünnschichtchromatographie ncben einer schnellen qualitativen Beurteilung vor allem auch eine quantitative Isolierung der Porphyrine als Methylester ermöglicht $(11,12)$. Die Vorteile des Verfahrens liegen in der Gewinnung von Reinsubstanzen und demzufolge in einer vereinfachten spektrophotometrischen Analyse. Unsere Versuche, Porphyrine als Vergleichssubstanzen aus porphyrischen Urinen zu isolieren, ergaben, daß sich diese Methode zur quantitativen Bestimmung von Copro- und Uroporphyrin im Urin eignet.

\section{Methodik}

\section{Reagenzien}

Mcthanol $/ \mathrm{H}_{2} \mathrm{SO}_{4} 5$ Vol-proz. ( $5 \mathrm{~m} / \mathrm{konz}$. $\mathrm{H}_{2} \mathrm{SO}_{4}$ in $95 \mathrm{~m} / \mathrm{Mcthanol}$ p. a. unter Kühlung); Natriumbicarbonat; Natriumsulfat; Petroläther (Kp. 30-60'); Benzol; Äthylacetat; Methanol; n-Butanol; Chloroform.

\section{Apparate}

Hochvakuumpumpe.

Rotationsverdampfer (,, Rotavapor" Fa. Büchi, Schweiz).

Dünnschichtchromatographic (Strcichgerät der Fa. Dcsaga, Hcidelbcrg): Glasplatten $20 \times 20 \mathrm{~cm}$, beschichtet mit Kicselgel $\mathrm{H}$ nach StAHL (Fa. E. Mcrck, Darmstadt), Schichtdicke 0,5 mm; Aktivierung der Platten nach $2 \mathrm{Stdn}$. Lufttrocknung bei $105^{\circ}$ über $60 \mathrm{Min}$. in Wärmeschrank.

UV-Lampe (355 nm; Quarzlampen-Gescllschaft m. b. H., Hanau). Schichtabsaug- und Elutionsgerät (Fritte G3) nach GoLDRrCK und Hirscri (13) der Fa. Glastechnische Werkstätten E. Zimmermann, Köln-Lindenthal, Joseph-Stelzmann-Str. 52.

Photometer: Zeiss Spektralphotometer PMQ II.

\section{Ausführung}

Lyoplsilisierung, Veresterung und Extraktion

10 (20) $\mathrm{m} /$ Urin werden in einen $100 \mathrm{~m} /$ Erlenmeycrkolben abgefüllt und in der Speedivac-Gefriertrocknungsanlage (Modell $5 \mathrm{PS}, \mathrm{Fa}$. Edwards High Vakuum) lyophilisiert. Alle Arbeiten crfolgen weitgehend im Dunkeln; die Gefäße werden mit schwarzem Papier umhüllt. Die gefriergetrockneten Proben werden mit 10 (20) $\mathrm{m} / \mathrm{Methanol}-\mathrm{H}_{2} \mathrm{SO}_{4}$ versctzt und 24 Stdn. bei Raumtempcratur gchalten. Während diescr Zeit crfolgt die Vercstcrung der freien Porphyrine zu Porphyrinmethylestern. Nach Zugabe von $50(100) \mathrm{ml}$ aqua dest. werden dic Porphyrinmethylester vicrmal mit 5-10 ml Chloroform kxäftig ausgeschüttelt. Der Chloroformextrakt wird einmal mit halbgesättigter wäßr. $\mathrm{NaHCO}_{3}$ und zwcimal mit aqua dest. gewaschen, über $\mathrm{Na}_{2} \mathrm{SO}_{4}$ getrocknct, in einen $25(50) \mathrm{m} l$ Spitzkolben filtricrt und am Rotationsverdampfer eingedampft.

\section{Dimmscbicbtcljromatograpljie}

Die in möglichst wenig Chlorofurm $(<0,5 \mathrm{~m} /)$ wieder gelösten Porphyrinmethylester werden auf eine Kieselgel-H-Platte in Form eincs dünnen Streifens, der sich über dic ganze Breitc der Platte erstreckt, mit ciner Saughütchen-Pipettc (ausgezogenes Glasrohr) aufgetragen. Sorgfältiges, mehrmaliges Nachspülen mit Chloroform und wiederholtes Auftragen auf dic Startzone sind für cine quantitative Ubbrführung unerläßlich. Einc dabci auftretende Verbrciterung des Startstrcifens wird nach kurzer Laufstrecke im ersten Lösungsmittelsystem ausgeglichen. Es laufen jeweils 'T'estsubstanzen (Protoporphyrin-IX-dimethylester, Coproporphyrin-I-tetra-methylester, Uroporphyrin-I-okta-methylester, Fa. KochLight-Laboratorics, Colnbrook, England) mit. Dic Entwicklung des Chromatogramms erfolgt aufstcigend in den Lösungsmittelsystemen:

A) Chloroform/Methanol 9:1 (V/V); Laufstrceke $2 \mathrm{~cm}$ (2 Min.), B) Petroläther/Äthyläther $1: 1$ (V/V); Laufstrccke $12 \mathrm{~cm}$ (20 Min.), C) Benzol/Äthylacetet/Methanol/Butanol $82: 14: 3: 1$ (V/V); Laufstrecke $10 \mathrm{~cm}$ (20 Min.).

Man trocknet zwischen den einzclnen Entwicklungsstufen die Platte mit einem Föhn. Mit dem System B werden nicht dic Porphyrine, sondern andere, im Chloroformextrakt enthaltene Harnfarbstoffe soweit aufgetrennt, daß3 sie im Lösungsmittelsystem $\mathrm{C}$ mit den $R_{\mathrm{F}}$-Werten der Porphyrinmethylester nicht interferieren. Nach Entwicklung der Platte im Lösungsmittelsystem $C$ werden die im UV-Licht rotfluoreszierenden Strcifen auf der noch feuchten Platte mit dem Skalpell markicrt, che dic Schicht diescr Substanzstrcifen nach Lufttrocknung der Platte mit dem Spatel abgelöst und in das Schichtabsaug- und Elutionsgerät, das an die Wassurstrahlpumpe angeschlossen ist, aufgenommen wird. Nach Elution der Coproporphyrinmethylester mit Chloroform, der Uroporphyrinmethylester mit Chloroform/Methanol 9:1 (V/V) unter Stickstoff werden dic Substanzen einzeln im L_ösungsmittelsystem C rechromatographiert (Abb. 1). Der Substanzverlust bei cincr chromatographischen Aufarbeitung im Bercich von 2-4 $\mu \mathrm{g}$ licgt für Uroporphyrin bei $3 \%$, für Coproporphyrin bci $5 \%$.
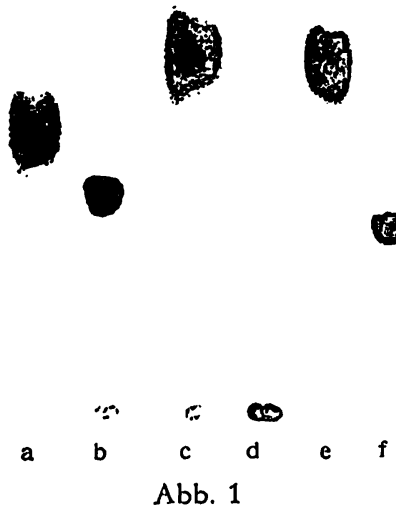

Kieselgel-H-Dünnschichtchromatogramm der Porphyrinmethylester (PME): a) Pentacarboxy-PME; b) Uro-PME: c) CoproPME; d) Protoporphyrin-IX-ME; c) Copro-PME; f) Uro-PME. a)-c) aus Urin isoliert, d)-f) Rcferenz-Substanzen. Lösungsmittclsystem: Benzol/Äthylacctat/Mcthanol/Butanol 82:14:3:1 (V/V). Aufnahme unter UV-Licht $(355 \mathrm{~nm})$.

\section{Spektroplotometriscbe Bestimmun!g}

Nach Abdampfen des Lösungsmittcls löst man dic Porphyrinmethylester in 2,5 oder $10 \mathrm{~m} /$ Chloroform und mißt dic Extinktion am Soret-Maximum (1). Mittels des millimolaren Extinktionskoeffizienten (Tab. 1) wird der Porphyringehalt berechnet: 
Tab. 1

Absorptionsmaxima der Porphyrinmethylester in Chloroform

\begin{tabular}{|c|c|c|c|c|c|c|c|c|}
\hline \multirow[t]{2}{*}{ Maxima } & \multicolumn{2}{|c|}{$\begin{array}{l}\text { Protoporphyrin-IX- } \\
\text { dimethylester }\end{array}$} & \multicolumn{3}{|c|}{$\begin{array}{c}\text { Coproporphyrin-I(III)- } \\
\text { tetramethylester }\end{array}$} & \multicolumn{3}{|c|}{$\begin{array}{l}\text { Uroporphyrin-I(III I)- } \\
\text { oktamethylester }\end{array}$} \\
\hline & $\mathbf{a}$ & $\mathbf{b}$ & $\mathbf{a}$ & b & c & $\mathbf{a}$ & b & c \\
\hline $\begin{array}{c}\text { Soret } \\
\text { IV } \\
\text { III } \\
\text { II } \\
\text { I }\end{array}$ & $\begin{array}{l}407 \\
505 \\
541 \\
575 \\
630\end{array}$ & $\begin{array}{l}408 \\
505 \\
540 \\
576 \\
625\end{array}$ & $\begin{array}{l}400 \\
498 \\
532 \\
566 \\
621\end{array}$ & $\begin{array}{l}399 \\
498 \\
532 \\
564 \\
619\end{array}$ & $\begin{array}{l}399 \\
498 \\
532 \\
564 \\
623\end{array}$ & $\begin{array}{l}406 \\
502 \\
536 \\
572 \\
627\end{array}$ & $\begin{array}{l}405 \\
500 \\
534 \\
570 \\
624\end{array}$ & $\begin{array}{l}405 \\
500 \\
534 \\
570 \\
624\end{array}$ \\
\hline$\varepsilon \mathrm{mMI}$ & \multicolumn{2}{|c|}{171} & \multicolumn{3}{|c|}{180} & \multicolumn{3}{|c|}{215} \\
\hline Mol.-Gew. (freie Säuren) & \multicolumn{2}{|c|}{562,64} & \multicolumn{3}{|c|}{654,69} & \multicolumn{3}{|c|}{830,73} \\
\hline
\end{tabular}

a und $\varepsilon_{\text {nII }}$ : nach FALK (1); b: Substanzen von Koch-Light-Laboratories (I-Isomere); c: aus Urin isolierte Porphyrine (III-Isomere) Messung von b und c: Recording Spectrophotometer CARY M 15 (Fa. „Applied Physic Corporation“", Monrovia, California).

Copro-(Uro-)porphyrinausscheidung $=$

$$
\frac{\mathrm{E} \cdot \text { Mol.-Gew. } \cdot \mathrm{V}_{1} \cdot \mathrm{V}_{3}}{\varepsilon_{\mathrm{mM}} \cdot \mathrm{d} \cdot \mathrm{V}_{2}}(\mu \mathrm{g} / \mathrm{Tag})
$$

$\varepsilon_{\mathrm{mM}}=$ millimolarer Extinktionskoeffizient

$\mathrm{d}=$ Schichtdicke (cm)

$\mathrm{V}_{1}=$ Volumen Urinausscheidung/Tag $(\mathrm{m} l)$

$\mathrm{V}_{2}=$ Volumen Urin zur Analyse $(\mathrm{m} l)$

$\mathrm{V}_{3}=$ Meßvolumen Chloroform (ml)

Die aus dem Urin isolierten Porphyrine werden anhand des $R_{F^{-}}$ Wertes im Vergleich zu Testsubstanzen durch Rotfluoreszenz im UV-Licht und mit spektrophotometrischer Analyse der Absorptionsmaxima (Abb. 2, Tab. 1) identifiziert. Damit erscheint die Spezifität der Methode gesichert.

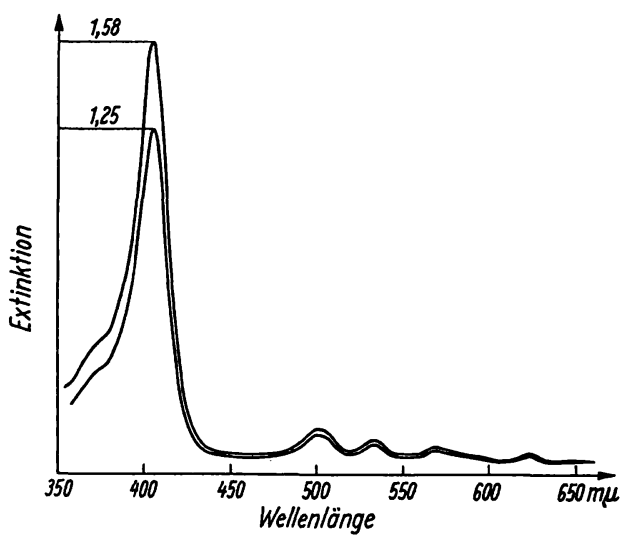

Abb. 2

Absorptionsmaxima von Uroporphyrin-I-oktamethylester $\left(\mathrm{E}_{405}=\right.$ 1,58 ; obere Kurve) und Uroporphyrin-III-oktamethylester $\left(\mathrm{E}_{405}=\right.$ 1,25; aus Urin isoliert) in Chloroform; Meßgerät: „Cary M 15“Spektrophotometer

Tab. 2

Rückgewinnungsversuche für Uro- und Coproporphyrin-ME; Zugabe der Substanz vor Extraktion der Porphyrin-ME aus Methanol/ $\mathrm{H}_{2} \mathrm{SO}_{4}$

\begin{tabular}{llccc}
\hline $\begin{array}{c}\text { Porphyrin- } \\
\text { Methylester } \\
(M E)\end{array}$ & $\begin{array}{c}\text { in } \\
10 \mathrm{ml} \text { Urin }\end{array}$ & $\begin{array}{c}\text { Mu- } \\
\text { gesetzt }\end{array}$ & $\begin{array}{c}\text { nach } \\
\text { Zusatz }\end{array}$ & $\begin{array}{c}\text { Ausbeute } \\
\%\end{array}$ \\
\hline $\begin{array}{l}\text { Coproporphyrin- } \\
\text { tetra-ME }\end{array}$ & $3,70(3,96)$ & 4,07 & $6,67(7,02)$ & 86,6 \\
$\begin{array}{l}\text { Uroporphyrin- } \\
\text { okta-ME }\end{array}$ & $4,38(4,77)$ & 5,61 & $9,57(8,96 ; 9,36)$ & 91,3 \\
\hline
\end{tabular}

Rückgewinnungsversuche

Die Überprüfung der Rückgewinnung schließt Extraktion, Chromatographie und Elution ein. Die Veresterung (mit nicht nachgetrocknetem Methanol p. a.) ist unter den angegebenen Bedingungen praktisch vollständig. Dies ergab sich für Di-, Tetraund Oktacarboxyporphyrine aus Versuchen mit bakteriellen Präparationen; mit Protoporphyrin (Fa. Sigma, Chemical Com- pany, USA) wurde der Befund auch gravimetrisch bestätigt. Es wurden bekannte Mengen Copro- und Uroporphyrinmethylester mehreren Urinproben zugesetżt und Urinproben ohne Zusätze parallel aufgearbeitet. Die Ergebnisse der Rückgewinnungsversuche für Copro- und Uroporphyrin zeigt Tabelle 2.

Präzision der Methode

16 Einzelbestimmungen in einem porphyrischen Urin, wobei je 8 Urinproben von 5 und $10 \mathrm{~m} /$ zur Analyse kamen, wurden statistisch ausgewertet und die Standardabweichung der Einzelbestimmung (s) sowie die prozentuale Abweichung vom Mittelwert (Variationskoeffizient, V) errechnet. Die Fehlerbreite der Analysen geht aus Tabelle 3 hervor.

Isomerenanalyse

Zur Differenzierung der einzelnen Porphyrieformen werden die Porphyrine der Isomerenreihen I und III chromatographisch getrennt. Die akut intermittierende Form der hepatischen Porphyrie, die als häufigste Porphyrinkrankheit in 60 bis $70 \%$ aller Fälle auftritt (14), wird vor allem durch den Nachweis von Uroporphyrin III diagnostisch gesichert. Uroporphyrin I wird bei der kongenitalen Porphyrie des Menschen und des Rindes gefunden.

\section{Uroporphyrin-Isomere}

Die Uroporphyrin-Isomeren werden als Methylester auf Papier (Whatman No. 1 oder Schleicher und Schüll 2043b) mit der Dioxan-Methode nach FALK und BENson (15) getrennt. $\mathrm{Da}$ aus den vorangehenden Isolierungsgängen reine Substanzen gewonnen werden, kann auf die erste Entwicklung im System Chloroform-Kerosin verzichtet werden. Die Chromatographie erfolgt aufsteigend im System Kerosin/Dioxan 4:1 (V/V) auf Papierstreifen $18 \times 11 \mathrm{~cm}$. Uroporphyrin-III-oktamethylester (Testsubstanz und Präparate aus porphyrischen Urinen) wandert 2,5 bis $3,0 \mathrm{~cm}$ und setzt sich meistens klar und ohne Streifenbildung ab, wenn nicht mehr als $0,5 \mu \mathrm{g}$ aufgetragen werden. Dagegen beobachtet man bei Uroporphyrin-I-oktamethylester (Testsubstanz und Isolierung aus Aerobiern (11)) häufig eine vertikale streifige Ausziehung der am Startpunkt liegengebliebenen Substanz zur Position des Uroporphyrin-III-methylesters. Für die Auswertung unter dem UV-Licht müssen die Chromatogramme trocken sein (Trocknung 10. Min. bei $105^{\circ}$ ). Die kritischen Seiten der Isomerentrennung, wie sie FALK (1) diskutierte, haben Conford und BENSON (16) einer detaillierten quantitativen Studie unterzogen, die es ermöglicht, Fehlbeurteilungen zu vermeiden. Die 
Tab. 3

Porphyrinbestimmung im Urin bei einer hepatischen Porphyrie

\begin{tabular}{|c|c|c|c|c|c|c|c|}
\hline \multirow{2}{*}{$\frac{\begin{array}{c}\text { Porphyrin- } \\
\text { Methylester (ME) }\end{array}}{\text { Coproporphyrin-III-tetra-ME }}$} & \multirow{2}{*}{ 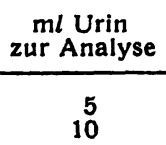 } & \multicolumn{4}{|c|}{$\begin{array}{c}\mu \mathrm{g} \text { Porphyrin-ME aus getrennt lyophillisierten } \\
\text { Urinproben (pro } 1000 \mathrm{ml} \text { Urin) }\end{array}$} & \multirow{2}{*}{$\frac{\bar{x} \pm 2 s}{346 \pm 35}$} & \multirow{2}{*}{$\begin{array}{r}V \\
\pm 5,0 \\
\pm 4,8\end{array}$} \\
\hline & & $\begin{array}{l}354 \\
350\end{array}$ & $\begin{array}{l}324 \\
332\end{array}$ & $\begin{array}{l}364 \\
358\end{array}$ & $\begin{array}{l}340 \\
322\end{array}$ & & \\
\hline Uroporphyrin-III-okta-ME & $\begin{array}{r}5 \\
10\end{array}$ & $\begin{array}{l}876 \\
890\end{array}$ & $\begin{array}{l}954 \\
838\end{array}$ & $\begin{array}{l}930 \\
868\end{array}$ & $\begin{array}{l}856 \\
910\end{array}$ & $\begin{array}{l}904 \pm 91 \\
877 \pm 62\end{array}$ & $\begin{array}{l} \pm 5,1 \\
\pm 3,5\end{array}$ \\
\hline
\end{tabular}

Uroporphyrinmethylester I und III lassen sich aber auch auf Dünnschicht-Cellulose-Platten (Cellulose-Pulver MN 300, Teilchengröße: $\varnothing 10 \mu$; Fa. Macherey-Nagel, Düren) $10 \times 8 \mathrm{~cm}$ in demselben Lösungsmittelsystem trennen. Der Trenneffekt ist gegenüber der Papiermethode unverändert.

\section{Coproporphyrin-Isomere}

Zur Trennung der Coproporphyrin-Isomeren erwies sich die aufsteigende Chromatographie der freien Säuren auf Papier nach ERIKSEN (1), die auf frühere Beobachtungen von FALK und Benson (1) zurückgeht, als am besten reproduzierbar. Mit einer Modifikation von JENSEN (17) können die Coproporphyrin-Isomere auch dünnschichtchromatographisch auf Kieselgel $G$ (wir verwendeten Kieselgel $\mathrm{H}$ ) getrennt werden.

Hydrolyse der Coproporpbyrinmetbylester: Man löst Porphyrinmethylester in $25 \mathrm{proz}$. $\mathrm{HCl}$; die Hydrolyse ist nach 48 Stdn. bei Raumtemperatur vollständig. Die Lösung wird mit $\mathrm{NaOH}$ und Natriumacetat auf $\mathrm{pH} 4$ gebracht; nach Extraktion der freien Säuren in Äther wird der Ätherextrakt mit aqua dest. neutral gewaschen, über einer kleinen Menge Natriumsulfat (2 Spatelspitzen) getrocknet, filtriert und eingedampft. Die freien Coproporphyrine werden in folgendem Lösungsmittelgemisch (ERIKSEN) aufgetragen: konz. Ammoniak/ Wasser/Aceton 1:2:7 (V/V). Die Entwicklung erfolgt auf Papierstreifen (Schleicher und Schüll 2043b) $18 \times 11 \mathrm{~cm}$ im Lösungsmittelsystem 2,6-Lutidin/Wasser $5: 2(\mathrm{~V} / \mathrm{V})$ oder auf Kieselgel-H-Platten $5 \times 20 \mathrm{~cm}$ im Lösungsmittelsystem 2,6-Lutidin/Wasser $10: 3$ (V/V). In beiden Verfahren wird zur atmosphärischen Sättigung ein zylindrisches Glas mit konz. $\mathrm{NH}_{4} \mathrm{OH}$ auf den Boden des Chromatographiegefäßes gestellt. CoproporphyrinIII ist die schneller wandernde Substanz.

\section{Ergebnisse und Diskussion}

\section{Uinuvandlung von Porpbobilinogen zu Uroporpbyrin}

Porphobilinogen kann im sauren Milieu oder enzymatisch zu Uroporphyrin III konvertieren. Die größte Menge von Uroporphyrin III (und geringe Mengen von Uroporphyrin I), die im Urin von Patienten mit akuter Porphyrie gefunden wird, entsteht nicht-enzymatisch aus dem ausgeschiedenen Porphobilinogen (1). Die idealen Bedingungen für diese chemische Konversion wurden noch nicht definiert (2). SchwARTZ und Mitarbeiter (2) erhielten die besten Ergebnisse bei Ansäuern des Urins auf $0,03 \mathrm{~N} \mathrm{HCl}$ und $30 \mathrm{Min}$. Erhitzen. im Wasserbad. Parallel dazu wurde der unbehandelte Urin aufgearbeitet, um den nativen Gehalt an Uroporphyrin zu bestimmen. Wir haben den Urin sofort (Einzelportionen oder nach dem Zeitpunkt des Sammelns) lyophilisiert oder bei $-20^{\circ}$ aufbewahrt, da wir im Urin, der sechs Tage bei $+4^{\circ}$ stand, eine Zunahme von Uroporphyrin feststellten. Sollen dagegen alle Intermediärprodukte (von einschließlich des Porphobilinogens bis einschließlich des Uroporphyrinogens), die zu Uroporphyrin konvertieren können, mit erfaßt werden, ist der Urin vorher zu erhitzen (2).

\section{Kritische Aspekte der Methode}

Ein Verfahren zur spektrophotometrischen Porphyrinbestimmung ohne Korrekturfaktoren muß notwendigerweise auf der Isolierung von Reinsubstanzen basieren. Die Porphyrinmethylester sind charakterisiert, wenn nach Chromatographie auf Kieselgel ein im UV-Licht rotfluoreszierender Fleck obne begleitende, andersfarbig fluoreszierende Zonen vorhanden ist, und die Lage der einzelnen Absorptionsmaxima (Tab. 1) und der Kurvenverlauf des Spektrogramms (Abb. 2) quiscben den einzelnen Absorptionsmaxima mit den ebenfalls dünnschichtchromatographisch gereinigten Vergleichssubstanzen identisch sind. Die Größenordnung der Ausbeuten und Abweichungen (Tab. 2,3) gewährt bei einer sicheren Kontrolle und Reproduzierbarkeit der chromatographischen Technik auch die Auswertung von Einzelbestimmungen (11).

Die beschriebene Methode wurde mit anderen Verfahren $(2,3,6,8)$ nicht systematisch verglichen, jedoch ergaben Stichproben, daß auch in fraglichen Fällen die Isolierung der Porphyrine gelang. Werden $10 \mathrm{ml}$ Urin aufgearbeitet, liegt der untere Bereich der Porphyrinbestimmung bei $36 \mu \mathrm{g}$ Copro- bzw. $39 \mu \mathrm{g}$ Uroporphyringehalt pro $1000 \mathrm{ml}$ Urin für eine Extinktion von 0,1 am SoretMaximum $\left(\mathrm{V}_{3}=2 \mathrm{ml} ; \mathrm{d}=2 \mathrm{~cm}\right)$. In $50 \mathrm{ml}$ gefriergetrocknetem Normalharn wurde der Coproporphyringehalt von $15 \mu \mathrm{g} / \mathrm{Tag}$ ( $1500 \mathrm{ml}$ Gesamtvolumen) erfaßt $(\mathrm{E}=0,14)$.

Auf die Isolierung und Bestimmung von Protoporphyrin wurde in der Methodik nicht eingegangen, da es bis auf $z$ wei Mitteilungen $(18,19)$ keine sicheren Hinweise für das Vorkommen von Protoporphyrin im normalen und pathologischen Harn gibt (2). In Analogie zum Nachweis bakterieller Dicarboxyporphyrine $(9,12)$ ist anzunehmen, daß Protoporphyrin aus dem porphyrischen Urin herausfraktioniert werden kann, wenn es vorliegt. Wir haben im Urin von acht an einer Porphyrie erkrankten Patienten kein Protoporphyrin nachweisen können. Ist im Porphyringemisch das relativ instabile Protoporphyrin enthalten, so wird die Veresterung bei $+4^{\circ}$ über $48 \mathrm{Stdn}$. durchgeführt (9), da im Vergleich der Protoporphyrinausbeuten aus bakteriellen Zellsuspen- 
sionen, in Abhängigkeit von Zeit und Temperatur, in den bei Zimmertemperatur veresterten Ansätżen um $20 \%$ weniger Protoporphyrin gefunden wurde. Protoporphyrin allein wird auch bei $-20^{\circ}$ in $36 \mathrm{Stdn}$. quantitativ in Methylester überführt. Die Ausbeute für Protoporphyrin (Extraktion, Chromatographie) beträgt bei $1 \mu \mathrm{g} 60 \%$, bei $10 \mu \mathrm{g} 70 \%$ und bei 5-10 mg (einschließlich Veresterung) gravimetrisch bestimmt über $80 \%$.
Die Verfasser sind Dr. Amy Benson, Department of Chemical Pathology, University College Hospital Medical School, London, und Dr. R. J. PorrA, C. S. I. R. O., Division of Plant Industry, Canberra (Australia) für die Úberlassung von Uroporphyrin-III Testsubstanz, Dr. June Lascelles, Department of Bacteriology, University of California, Los Angeles, für die Úberlassung von Coproporphyrin-III Testsubstanz zu Dank verpflichtet.

Die Messung der Absorptionsmmaxima wurde mit freundlicher Unterstützung durch Herrn Prof. Dr. H. Wrtzes, Biochemische Abteilung des Chemischen Instituts der Universität Marburg, durchgeführt.

\title{
Literatur
}

1. FALK, J. E., Porphyrins and Metalloporphyrins, Elsevier, Amsterdam-London-New York (1964). - 2. Schwartz, S., M. H. Berg, I. Bossenmater und H. Dinsmore, Methods of Biochemical Analysis, Bd. VIII, Interscience, New York (1960). - 3. SCHLENKer, F. S. und C. L. Krtchell, Amer. J. Clin. Path. 29, 593 (1958). -4. Schlenker, F. S., N. A. Taylor und C. L. Kitchell, Amer. J. Clin. Path. 44, 189 (1965). - 5. Franzini, C. und M. L. SolA, Minerva med. 57, 112 (1966). - 6. Rimington, C. und S. L. Svernsson, Scand. J. Clin. Laborat Invest. 2, 209 (1950). - 7. Rimington, C., Biochem. J. 75, 620 (1960). - 8. Wirh, T. K., Scand, J. Clin. Laborat. Invest. 7, 193 (1955). - 9. Doss, M. und
W. Mannherm, Experientia (Basel), 23, 31 (1967). - 10. Demole, E., J. Chromatogr. (Amsterdam), 1, 24 (1958). - 11. Mannherm, W. und M. Doss, Z. Naturforsch. 22b, 359 (1967). - 12. MANNHRIM, W. und M. Doss, in Vorbereitung. - 13. GoldRICK, B. und J. Hrrsch, J. Lipid Res. 4, 482 (1963). - 14. Filippini, L., Dtsch. med. Wschr. 91, 959 (1966). - 15. FALK, J. E. und A. Benson, Biochem. J. 55, 101 (1953). - 16. Conford, P. A. D. und A. BENson, J. Chromatogr. (Amsterdam) 10, 141 (1963). — 17. JENSEN, J., J. Chromatogr. (Amsterdam) 10, 236 (1963). - 18. BrugsCH, J., Zschr. exper. Med. 103, 518 (1938). - 19. Watson, C. J., J. Clin. Invest. 15, 327 (1936).

\section{Quantitative Erfassung und klinische Bedeutung der Betaninurie}

\author{
Von M. Geldmacher-v. Mallinckrodt, M. Тң. Arello und M. V. Arelloº) \\ Aus dem Institut für gericbtliche Medizin und Kriminalistik der Universität Erlangen-Niirnberg \\ (Direktor: Prof. Dr. Dr. E. Weinig)
}

(Eingegangen am 17: Februar 1967)

\begin{abstract}
Es ist seit langem bekannt, daß gelegentlich nach Genuß von Roten Rüben (Beta vulgaris) rot gefärbter Ưrin ausgeschieden wird. Dies kann den Verdacht einer Hämaturie oder der Ausscheidung bestimmter Medikamente erwecken. Bei dem ausgeschiedenen roten Farbstoff handelt es sich um Betanin. Während bisher angenommen wurde, daß nicht alle Personen „Ausscheider" sind, sondern das Phänomen abhängig sei vom pH-Wert des Urins, von genetischen Gegebenheiten, Permeabilitätsstörungen der Niere, allergischen Erscheinungen, bestimmten Anämieformen oder auch dem Farbstoffgehalt der Roten Rüben bzw. der aufgenommenen Rübenmenge, konnten wir bei allen untersuchten gesunden Personen mit einem empfindlichen säulenchromatographischen Verfahren nach Rote Rüben-Aufnahme Betanin, wenn auch z. T. in sehr geringer Menge im Harn nachweisen. Bei ein- und derselben Person zeigten sich vón Tag zu Tag starke Schwankungen sowohl bezüglich der ausgeschiedenen Menge als auch der Konzentration. Tägliche Aufnahme über 47-66 Tage führte zu keiner nennenswerten Änderung etwa im Sinne einer Fermentadaptation. Damit läßt sich die Einteilung in Betanin-,,Ausscheider" und -,,Nichtausscheider" nicht aufrechterhalten, die Fähigkeit zur Resorption und Ausscheidung von Betanin scheint vielmehr grundsätzlich gegeben zu sein, wenn auch quantitative Unterschiede auftreten. Selbst bei stärkerer Ausscheidung von Betanin im Harn konnte im Serum Betanin
\end{abstract} nicht nachgewiesen werden.

It has been known for a long time that the urine may sometimes become red after the ingestion of beetroot (Beta vulgaris). This recalls the condition of hematuria or the excretion of certain drugs. The excreted red pigment is betanine. It has been hitherto assumed that not all persons are "excreters", but that the phenomenon depends on the $\mathrm{pH}$ of the urine, genetic predisposition, faulty permeability in the kidneys, allergy, certain forms of anaemia, or even the pigment content of the beet or the amount of beet eaten. With the aid of a sensitive column chromatographic procedure, however, we have detected betanine, albeit sometimes in very small amounts, in the urine of all investigated healthy persons after the ingestion of beet. Each individual shows marked variations from day to day in both the amount and concentration of excreted pigment. Daily ingestion for 47-66 days caused no significant changes, and there was no indication of enzyme adaptation. Thus the classification into betanine "excreters" and "non-excreters" is false; the ability to absorb and excrete betanine appears to be subject to more fundamental factors, even though there are quantitative variations. Betanine could not be detected in the serum, even when the urinary betanine excretion was very high.

\section{Betanin im Harn}

Es ist seit langem bekannt, daß manche Personen nach Genuß von Roten Rüben (Beta vulgaris) rot gefärbten Urin ausscheiden. Dies Phänomen kann den Verdacht

1) Die Arbeit wurde mit Hilfe der Deutschen Forschungsgemeinschaft durchgeführt, wofür wir auch an dieser Stelle unseren besten Dank aussprechen. einer Hämaturie oder einer Aufnahme bestimmter Medikamente erwecken. Von vornherein wurde vermutet, daß die Rotfärbung durch Betanin, den Farbstoff der Roten Rüben, verursacht wird.

Die Konstitution von Betanin ist erst 1965 durch die Arbeitsgruppe um WYLER und DrEIDING (1) endgültig geklärt worden. Danach handelt es sich nicht - wie 